\section{Lymphangioma as a rare cause of acute recurrent pancreatitis}

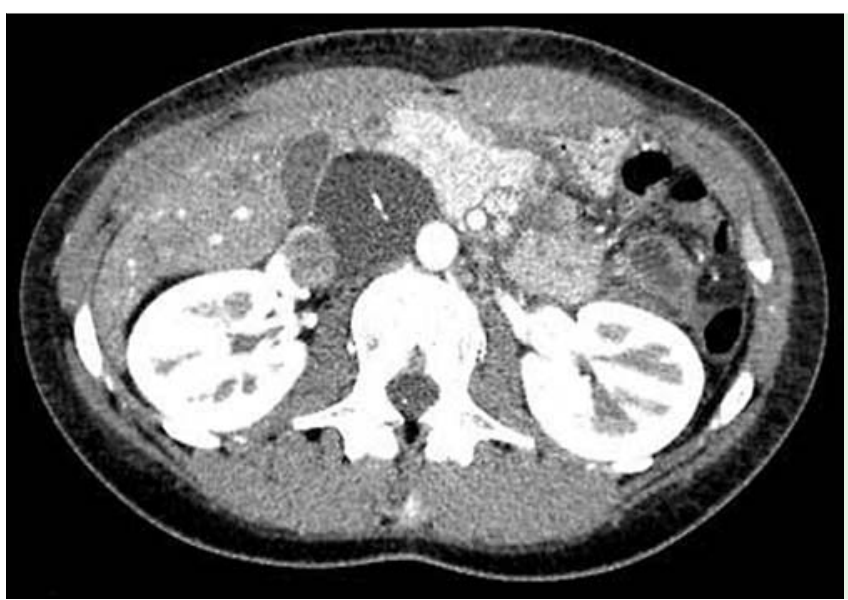

Fig. 1 Lymphangioma causing acute recurrent pancreatitis. Computed tomography shows a cystic mass behind the pancreatic head with thin calcifications and lipid-like tissue.
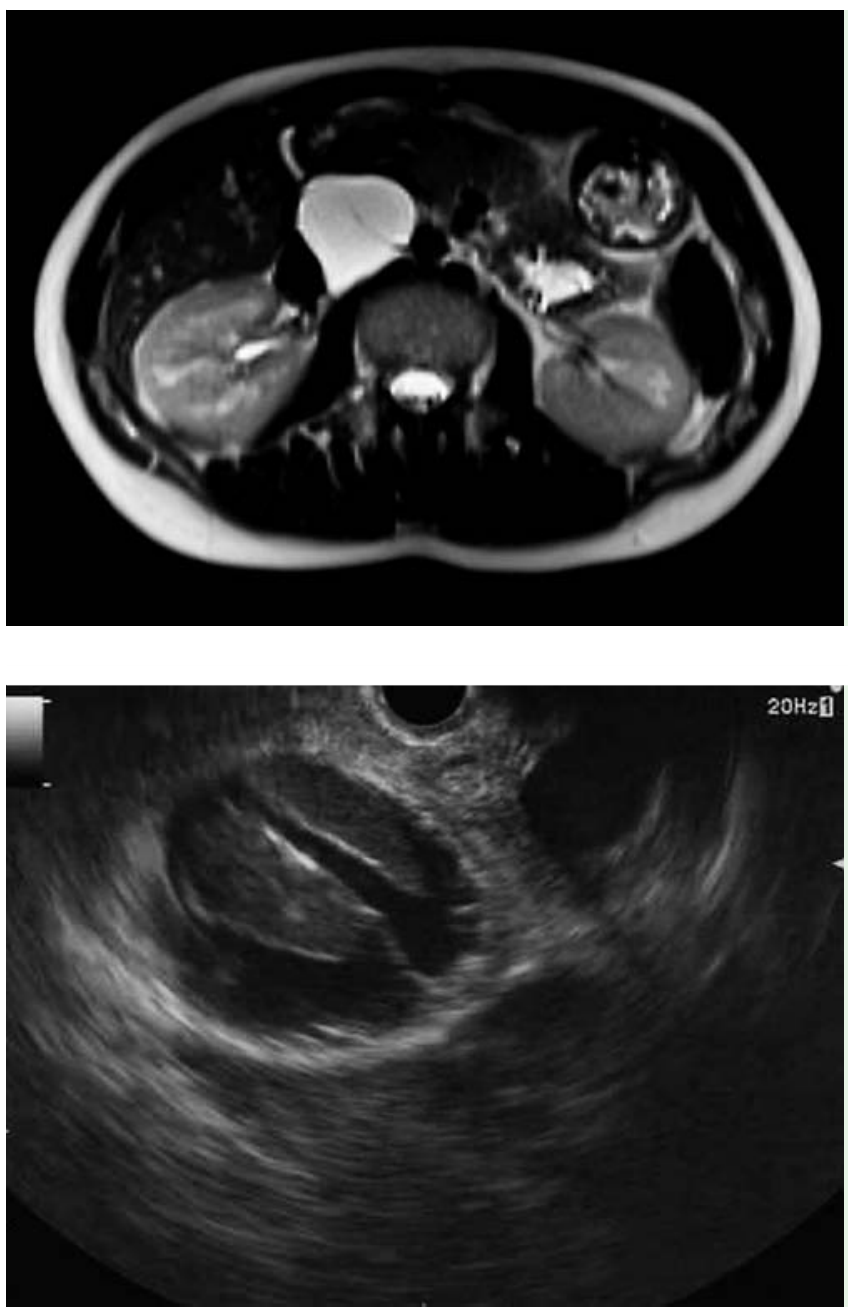

A 26-year-old woman presented with sharp pain in the upper abdomen. Elevated amylase and lipase levels suggested pancreatitis. Emergency computed tomography (CT) of the abdomen showed a cystic lesion with calcifications and lipidlike tissue located between the aorta and vena cava and compressing the pancreatic head and duodenum ( $\bullet$ Fig. 1). Teratoma was suspected. The patient had experienced acute idiopathic pancreatitis 4 years earlier, at which time CT had shown a pancreatic head pseudocyst, so it seemed that the appearance of the lesion had changed.

The patient was referred for magnetic resonance imaging, which showed a cystic lesion with a thin capsule, septa, calcifications, and a solid part clearly not originating from the pancreatic head ( $\bullet$ Fig. 2). The patient then underwent endoscopic ultrasound (EUS) because of the suspicion of teratoma. EUS showed a well-delineated cystic lesion with hyperechoic septa and homogeneous, more solid parts. Calcifications were not seen on EUS ( $\bullet$ Fig.3). EUS-guided fine-needle aspiration was performed with a 22-gauge needle (Expect; Boston Scientific, Natick, Massachusetts, USA). Chylous, milky white fluid was aspirated from the cyst. Biochemistry of the fluid showed a high level of triglycerides and low levels of carcinoembryonic antigen (CEA) and amylases, which definitely excluded pseudocyst from the differential diagnosis. Sediments of the fluid contained lymphocytes that were CD3+ on immunocytochemistry. The cytologic diagnosis was consistent with cystic lymphangioma. The patient refused surgical treatment. On follow-up, she was symptoms free and had serum values of amylase, lipase, cancer antigen (CA) 19-9, and CEA within normal range.

This case is interesting because it shows lymphangioma as a rare cause of recurrent acute pancreatitis. Lymphangioma is a malformation of lymphatic vessels and should not be misinterpreted as a cystic or solid-cystic pancreatic tumor $[1,2]$. Although it is benign, a compression effect on other organs can cause symptoms [3]. The patient had no other probable cause of acute pancreatitis, and we therefore concluded that in this case lymphangioma was the cause of pancreatitis.

Endoscopy_UCTN_Code_CCL_1AF_2AF_3AC

Competing interests: None 
Mario Tadic', Zeljko Cabrijan', Tajana Stoos-Veic ${ }^{2}$, Mirjana Vukelic-Markovic ${ }^{3}$

${ }^{1}$ Department of Gastroenterology, Dubrava University Hospital, Zagreb, Croatia

${ }^{2}$ Department of Pathology and Cytology, Dubrava University Hospital, Zagreb, Croatia

${ }^{3}$ Department of Radiology, Dubrava University Hospital, Zagreb, Croatia

\section{References}

1 Sriram PV, Weise C, Seitz $U$ et al. Lymphangioma of the major duodenal papilla presenting as acute pancreatitis: treatment by endoscopic snare papillectomy. Gastrointest Endosc 2000; 51: $733-736$

2 Coe AW, Evans J, Conway J. Pancreas cystic lymphangioma diagnosed with EUS-FNA. JOP 2012; 13: 282-284

3 Black T, Guy CD, Burbridge RA. Retroperitoneal cystic lymphangioma diagnosed by endoscopic ultrasound-guided fine needle aspiration. Clin Endosc 2013; 46: 595-597

\section{Bibliography}

Dol http://dx.doi.org/ 10.1055/s-0034-1390720

Endoscopy 2014; 46: E598-E599

(c) Georg Thieme Verlag KG

Stuttgart · New York

ISSN 0013-726X

\section{Corresponding author}

Mario Tadic, MD, PhD

Department of Gastroenterology Dubrava University Hospital

Av. Gojka Suska 6

10040 Zagreb

Croatia

Fax: +38512902550

mtadic1@gmail.com 